\title{
Demanda y Consumo de Productos Orgánicos en el Cantón Riobamba, Ecuador
}

\author{
Claudia M. Andrade ${ }^{(1)}$ y Dante Ayaviri(2) \\ (1) Facultad de Recursos Naturales, Instituto de Posgrado y Educación Continua, Escuela Superior \\ Politécnica de Chimborazo, Riobamba-Ecuador. (e-mail: claudimari8873@yahoo.es) \\ (2) Facultad de Ciencias Políticas y Administrativas, Universidad Nacional de Chimborazo, Riobamba - \\ Ecuador. (e-mail: vdayaviri@gmail.com)
}

Recibido Dic. 1, 2017; Aceptado Feb. 9, 2018; Versión final Abr. 9, 2018, Publicado Ago. 2018

\begin{abstract}
Resumen
Se determina el nivel de consumo y demanda de productos orgánicos en la población urbana del Cantón Riobamba, Ecuador. Se emplearon técnicas de recolección de datos tales como entrevistas y encuestas aplicadas a una muestra de 384 personas a través de un muestreo aleatorio simple. Los principales lugares de compra de productos orgánicos son ferias especiales con frecuencia semanal donde los consumidores están dispuestos a pagar un precio más elevado por productos orgánicos versus productos convencionales. Las principales barreras para el crecimiento del consumo de productos orgánicos, son la baja oferta disponible, la falta de garantía de orgánicos, su corta vida útil y los precios elevados. Los principales productos orgánicos consumidos son verduras, hortalizas y frutas orgánicas. Como conclusión de la investigación se determinó que el nivel de ingresos y la escolaridad de la población no inciden sobre la demanda y consumo de productos orgánicos.
\end{abstract}

Palabras clave: productos orgánicos; verduras orgánicas; demanda y consumo

\section{Demand and Consumption of Organic Products in the Riobamba Cantón, Ecuador}

\begin{abstract}
The level of consumption and demand of organic products in the urban population of the Riobamba Canton, in Ecuador is determined and discussed. To generate empirical evidence, data collection techniques such as interviews and surveys applied to 384 people were done. The main place for purchasing organic products are special markets with a weekly frequency where consumers are willing to pay a higher price for these types of products compared to conventional products. The main barriers to the growth of the consumption of organic products are the low supply available, the lack of guarantee that the products are organic, the short life for their consumption and the high prices. The main organic products consumed are organic vegetables and fruits. As a conclusion of the research it is determined that the level of income and schooling of the population do not affect the demand and consumption of organic products.
\end{abstract}

Keywords: organic products; organic vegetables; demand and consumption 


\section{INTRODUCCION}

Los cambios ambientales percibidos en los últimos años como el calentamiento global, la contaminación por basura doméstica, las sequías, inundaciones y el incremento de plagas y enfermedades en los cultivos, han obligado al sector agrícola a emplear técnicas de producción insostenibles debido a los impactos negativos al ambiente, como el uso excesivo de fertilizantes químicos y plaguicidas para garantizar el abastecimiento de alimentos en los mercados ante la creciente demanda de consumo. En este sentido (Cardozo, Rodríguez, y Guaita, 2011) indican que relativa escasez de ciertos recursos naturales, han hecho que se exploren nuevas fuentes de provisión de materia prima, nuevas formas de transformación y se diversifiquen los procesos productivos que en muchos casos generan un impacto ambiental negativo. A este modo de producción se conoce como sistema tradicional o convencional. Al respecto Cruz et al., (2010), indican que en la actualidad, diversos factores de carácter ambiental, social, económico, cultural y político, han motivado el interés por el desarrollo de la agricultura orgánica, reconociéndose como una alternativa económicamente eficiente, socialmente justa y ecológicamente sostenible con potencial para atenuar los impactos negativos atribuidos a la agricultura convencional.

La agricultura orgánica es una estrategia de desarrollo que trata de cambiar algunas de las limitaciones encontradas en la producción convencional. Más que una tecnología de producción, la agricultura orgánica es una estrategia de desarrollo que se fundamenta no solamente en un mejor manejo del suelo y un fomento al uso de insumos locales, pero también un mayor valor agregado y una cadena de comercialización más justa (Soto, 2003). La agricultura orgánica es un sistema de producción que mantiene y mejora la salud de los suelos, los ecosistemas y las personas. Se basa fundamentalmente en los procesos ecológicos, la biodiversidad y los ciclos adaptados a las condiciones locales, sin usar insumos que tengan efectos adversos (Cruz, Ángel, Rindermann, Rufino, y Tovar, 2010). Cabe señalar por ejemplo que el control de plagas, busca actuar sobre las causas de aparición de las plagas, y no sobre la población misma. Busca quebrar las condiciones ideales de alimentación, reproducción y crecimiento de las plagas y fomentar la protección de sus enemigos naturales (Soto, 2003).

Los productos orgánicos abarca a la producción de vegetales y animales, obtenidos sin el uso de substancias químicas (pesticidas, herbicidas, fertilizantes sintéticos) ni el uso de organismos genéticamente modificados (OGM), y que tienen un reconocimiento por tal manejo (Flores y Andrade, 2008). Los motivos para que los productores hagan la conversión de la agricultura convencional a la orgánica, se destaca la influencia de algún familiar o vecino, el beneficio económico de comercializar los productos en espacios diferenciados y el cuidado del medio ambiente, además del apoyo y asesoría de los productores y académicos, principalmente, durante el proceso de transformación de los cultivos (Rueda et al., 2016). En este sentido la agricultura orgánica constituye una actividad económica con potencialidad en la generación de empleo y divisas. Su adopción requiere $30 \%$ más de mano de obra por hectárea con respecto a la producción convencional (Cruz et al., 2010).

De acuerdo a (Bartels, Reinders, Sen, y Du, 2006)), la demanda de los consumidores de productos orgánicos ha crecido de manera espectacular en Estados Unidos. Las ventas de alimentos orgánicos en dicho país generaron aproximadamente 31.320 millones de dólares en 2012 y se prevé que alcanzó 42.000 millones de dólares en 2014. De acuerdo a los resultados de la investigación realizada por Diaz y Perez ( 2015), las variables que influyen en el consumo son: productos costosos, desconocimiento de los beneficios y difíciles de encontrar en el mercado, son preferidos a los productos convencionales por ser considerados productos saludables y general frescos, el consumidor los reconoce por el empaque y el sello de certificación, y las compras se realizan semanalmente. De acuerdo a Vázquez et al., (2012), el consumo de productos orgánicos se da por su beneficio para la salud (86\%), preocupación ambiental (31\%), sabor (26\%), frescura (20\%) y no tener residuos de agroquímicos (3\%). Además el mismo autor estima que el $61 \%$ de los consumidores estuvo dispuesto a pagar un $10 \%$ de sobreprecio por los productos orgánicos. En esta línea Bartels et al., (2006), indican que la compra de productos orgánicos ocurre actualmente entre una gama más amplia de datos demográficos, con el $81 \%$ de las familias estadounidenses, que consumen productos orgánicos con más frecuencia que otros.

En la agricultura ecológica, la implantación de sistemas de certificación ha sido necesaria no sólo porque, a diferencia de otros productos agroalimentarios de calidad, sus atributos no son distinguibles a simple vista, sino porque las características diferenciales que aportan los alimentos ecológicos no tienen ningún otro valor sino ser claramente percibidas y apreciadas por el consumidor ((Velleda Caldas, Anjos, y Lozano Cabedo, 2014). El éxito de cualquier proyecto de producción orgánica depende de una adecuada comercialización. Es importante el desarrollo de los mercados locales, para lograr un crecimiento sostenido de la producción orgánica (Soto, 2003). De acuerdo a (Diaz vazquez Antonio, Perez Hernandez Amalia, 2015), los precios de los alimentos y bebidas orgánicas en el mercado son mucho más elevados que los convencionales, este sobre precio varía según el punto de venta, que generalmente represente entre un 10 
y $20 \%$ más. En este mismo sentido Salazar et al., (2011), indica que el sobreprecio que se paga por la adquisición de productos orgánicos llega hasta el 300\%, menciona también que es importante incrementar y diversificar la oferta de alimentos orgánicos, para abastecer la creciente demanda tanto interna como global. El precio y los ingresos, en cierta medida, afectan las compras de productos orgánicos por parte de los consumidores (Smith et al., 2009).

En el 2011, la producción orgánica mundial utilizaba el 1\% de las tierras agrícolas, con la participación de 162 países y más de 5.000 productos o artículos orgánicos, con un crecimiento del mercado estimado en un 10,4\% anual. América Latina concentra el 17\% del total de productores orgánicos del mundo, donde México es el país que registró la mayor cantidad de productores orgánicos (169 570) reportados en el 2011, mientras que Argentina posee 3,8 millones de hectáreas (ha) certificadas (Camacho et al., 2015). El incremento en la demanda de productos orgánicos estará limitado en función de una mayor cultura de los beneficios del consumo de productos orgánicos respecto a la salud humana y conservación del ambiente y a una mayor capacidad de compra de los consumidores (Vázquez et al., 2012). En función de los antecedentes expuestos el objetivo de la presente investigación es determinar el nivel de consumo y demanda de productos orgánicos en la población del cantón Riobamba, Provincia de Chimborazo, Ecuador, para alcanzar un sistema de producción sostenible que permita alcanzar el buen vivir de la sociedad.

\section{MATERIALES Y METODOS}

La presente investigación corresponde a un trabajo no experimental bajo un diseño transversal de recolección de datos por medio de entrevistas y la aplicación de una encuesta, se asume un enfoque cualitativo y cuantitativo, en primer lugar se realiza la descripción del problema de estudio y luego se realiza un análisis cuantitativo de los hallazgos del trabajo de campo con un alcance descriptivo correlacional. El cantón Riobamba cuenta con 225.700 habitantes, el tamaño de la muestra para esta investigación es de 384 personas, con un nivel de confianza del 95\% y un error del 5\%. Para la aplicación de la encuesta se empleo como marco de muestreo la capital provincial, ya que es ahí donde los habitantes realizan actividades económicas, sociales y personales, se toma en consideración un rango de edad entre 14 y 65 años como variable demográfica para la selección de los elementos muéstrales de la población objetivo, para obtener la información de interés sobre la demanda y consumo de productos orgánicos. El muestro probabilístico permitió que las unidades muéstrales sean seleccionadas al azar, a través del muestro aleatorio simple. La técnica de investigación cualitativa aplicada fueron las entrevistas semiestructuradas aplicadas a los consumidores de las diferentes clases sociales de la población en las ferias especiales de productos orgánicos que se desarrollan en la ciudad. Con los datos e información recolectada en el trabajo de campo se procedió a realizar el análisis estadístico descriptivo y la aplicación de un modelo econométrico.

\section{RESULTADOS}

Los productos alimenticios orgánicos contribuyen de una manera más eficiente a la nutrición humana. Según Tobar et al., (2010), los alimentos denominados orgánicos, deben haber sido cultivados y procesados utilizando métodos de agricultura orgánica, la cual recicla productos y promueve la biodiversidad. Los productos deben crecer sin abonos sintéticos, variedades transgénicas, fertilizantes derivados del petróleo y otros agroquímicos. Los animales deben tener acceso al aire libre, no son suministradas hormonas o antibióticos y se deben alimentar con pastos en lugar de comida sintética. Los alimentos procesados o industrializados no utilizan aditivos, colorantes o conservadores artificiales (López et al., 2012).

En los resultados reportados por Salgado y Beltrán (2011), señalan que se ha confrontado los factores internos del consumidor (comportamiento y aspectos demográficos) que ejercen gran influencia sobre la toma de decisión del consumo de productos orgánicos. Específicamente, adquirir productos por un factor como es la salud, ha resultado tener mayor significancia que la conciencia ecológica como indicadores de este comportamiento de los consumidores. A su vez, la escolaridad (nivel de estudios) sigue siendo un indicador importante dentro de los aspectos demográficos aunque por sí solo no es generador de un consumo sustentable, pero presenta alta significancia. En este sentido se puede observar que el motivo del consumo de productos orgánicos es llevar un estilo de vida saludable, seguido por quienes consideran que son alimentos más saludables que los convencionales, y los consume porque consideran que protegen al medio ambiente, lo cual muestra que estos consumidores tienen un poco más elevado su sentido de consciencia ambiental (Moreira, 2016). Debe ponerse énfasis en el diseño de estrategias del precio en los productos orgánicos para conseguir una mayor capacidad de pago, el escaso conocimiento o familiaridad sobre el proceso de producción de este tipo de productos que tienen los consumidores reduce significativamente su capacidad de pago (Salgado y Beltrán, 2011). 
El Ecuador no ha sido la excepción en cuanto al crecimiento de la agricultura orgánica. Los primeros proyectos, impulsados por algunas Organizaciones no Gubernamentales (ONGs) u organizaciones de desarrollo, que establecieron como eje de intervención el apoyo a la agricultura orgánica datan de los años 90. A partir de ahí se experimentó un crecimiento importante en cuanto a la superficie dedicada a la producción de alimentos agrícolas orgánicos, principalmente para la exportación (Flores y Andrade, 2008).

\section{i) Descripción de la zona de estudio}

De acuerdo al censo de población y vivienda del Instituto Nacional de Estadísticas y Censos (INEC, 2010), el cantón Riobamba cuenta con 12 parroquias, representa el $15,1 \%$ del territorio de la provincia de Chimborazo, el $49,2 \%$ de la población de esta provincia vive en el cantón Riobamba y equivale a 225.700 habitantes, el $64,8 \%$ de esta población vive en el sector urbano y el $35,2 \%$ en el sector rural. El $52,7 \%$ de la población son mujeres y $47,3 \%$ son hombres, la población económicamente activa (PEA), equivale al $54,7 \%$ y corresponde al 50,3\% de la PEA de la provincia de Chimborazo.

El $21,5 \%$ de la población del cantón Riobamba se ocupa en la agricultura, ganadería, silvicultura y pesca, y el $19,1 \%$ se dedica al comercio al por mayor y menor entre las principales actividades económicas. En el año 2012 el Impuesto a la Renta recaudado en el cantón Riobamba represento el 91,5\% del total de la provincia de Chimborazo y respecto al año 2010 creció en 38,6\%. La pobreza en el cantón Riobamba medido por necesidades básicas insatisfechas (NBI), es de $34,4 \%$ con respecto a la provincia de Chimborazo. La escolaridad de la población en el cantón Riobamba es de 9,9 años para las mujeres y de 10,8 años para los hombres.

\section{ii) Análisis estadístico}

Dentro del análisis estadístico realizado se determina que el $25,8 \%$ de los encuestados corresponden al género masculino y el $74,2 \%$ al género femenino. La edad promedio de la muestra es de 38,94 años, con un rango de 14 a 65 años. Únicamente el 2,1\% de los encuestados tiene un nivel de educación primaria, el $13,3 \%$ nivel de educación secundaria, el $73.7 \%$ de la población tiene un nivel de educación universitaria, y el $10,9 \%$ de la población un nivel de educación de posgrado, estos resultados son bastante pertinentes ya que Riobamba es considerada como la ciudad universitaria del Ecuador. Así el nivel de escolaridad es una variable importante ya que se asocia a un mayor nivel de ingresos con un mayor nivel de educación. En este sentido el $7,6 \%$ de los encuestados tienen ingresos menores a los 300 dólares mensuales, el $22,7 \%$ tienen ingresos entre 300 y 600 dólares por mes, el $41,7 \%$ de la población tienen ingresos entre 601 y 900 dólares, y el $28,1 \%$ de los encuestados tienen ingresos mayores a 1000 dólares mensuales.

El $97,4 \%$ de los encuestados mencionan conocer que son los productos orgánicos y el $77,9 \%$ afirman que los consumen. En un estudio realizado en el ciudad de Quito-Ecuador por Vasco et al., (2015), muestran en sus resultados que las personas que poseen un mayor nivel de escolaridad así como más ingresos económicos, gastan más en el consumo de productos orgánicos y ecológicos. Por lo que recomienda fomentar y promover la producción ecológica a fin de reducir los costos y el precio final al consumidor, y difundir los beneficios y ventajas de consumir productos ecológicos entre los segmentos de la población con menos escolaridad e ingresos.

El $70,6 \%$ de la población indica que la principal razón por la que consume productos orgánicos es para mantener y mejorar su salud, el 18,2\% por cuidar el medio ambiente, y el 11,2\% por otras varias razones. Los motivadores de compra de productos orgánicos coinciden plenamente con las razones de compra por la población: salud y cuidado del ambiente. El $63 \%$ de la población indican que la característica principal para decidir la compra son los beneficios para la salud que otorgan los productos orgánicos y un $26,8 \%$ de los encuestados consideran la variable precio para decidir la compra de este tipo de productos. Dentro de los principales beneficios del consumo de productos orgánicos el $78,1 \%$ de la población considera que su consumo ayuda reducir la incidencia de enfermedades, y solo 5,7\% considera que contribuye a conservar el medio ambiente, lo cual afirma la preocupación de la presente investigación por aportar al crecimiento de la demanda de productos orgánicos en esta población con fines de preservar el medio ambiente a través de sistemas de producción agrícola sostenibles.

El $38,5 \%$ de la población indica que el principal lugar de compra de productos orgánicos son las ferias especiales, seguido por el $34,9 \%$ que indican que realizan sus compras en los mercados populares de la ciudad, y solo el $13,8 \%$ de la población adquiere productos orgánicos en el supermercado. Este hallazgo es de gran relevancia ya que solo una pequeña parte de la población compra productos orgánicos en los supermercados con la garantía de orgánicos y a un precio más elevado en comparación con las ferias especiales de productos orgánicos. En esta línea (Parrado y Montoya, 2007), indican que la población de mayores ingresos presentan las menores frecuencias de compra para la mayoría de vegetales pero muestran alta disposición a comprar los productos a domicilio especialmente productos orgánicos, y los de 
menores ingresos que tienen mayores frecuencia de compra para la mayoría de productos, no conocen qué son los vegetales orgánicos, biológicos o ecológicos y prefieren la tienda sobre el supermercado. El $44,8 \%$ de la población adquiere productos orgánicos una vez por semana, el 25,5\% cada 15 días, el 17,2\% con una frecuencia mensual.

El $81 \%$ de la población del cantón Riobamba estarían dispuestos a pagar un precio más alto por los productos orgánicos. El $59,1 \%$ de la población gasta mensualmente entre 1 y 25 dólares en la compra de productos orgánicos, el $25,5 \%$ entre 26 y 50 dólares y únicamente el 15,3\% de la población gasta mensualmente más de 50 dólares en la compara de productos orgánicos muy seguramente en los supermercados. El $32,3 \%$ de la población consideran importantes las redes sociales como medios de comunicación para incentivar el consumo de productos orgánicos, el $25 \%$ de los encuestados se enteran de la oferta de productos orgánicos por sus amigos que ya los consumen y la radio no deja de ser importante con un $17,7 \%$ de aceptación.

Dentro de los principales problemas que influyen en el consumo y demanda de productos orgánicos en la población del cantón Riobamba, el 23,4\% expresan que existe poca oferta, el 12,2\% que tienen precios elevados, el $8,9 \%$ mencionan que los productos que se venden actualmente como orgánicos no tienen la garantía del caso, el 7\% de la población considera que existe escasa difusión y pocos lugares de comercialización y el 2,6\% consideran que tienen corta vida útil. Este resultado concuerda con lo reportado por Barbosa et al., (2011), que indican que el principal problema mencionado por las personas cuestionadas acerca de las dificultades para consumir este tipo de producto fue el factor precio, seguido por su disponibilidad.

El 35,7\% de la población indican que el producto orgánico mas consumido son las verduras, seguido por el $24,7 \%$ que consumen hortalizas orgánicas, y el $21,9 \%$ que consume frutas también orgánicas, esto se relaciona con el desarrollo de ferias especiales para la venta de este tipo de productos que los hace más accesibles al consumidor ya que es lugar preferido por los Riobambeños para comprar este tipo de productos. En esta línea (Flores y Andrade, 2008), indican que los principales productos certificados como orgánicos en Ecuador son las frutas, los cereales y leguminosas, las hortalizas y las plantas aromáticas. Los principales cultivos orgánicos que cuentan actualmente con certificación son el banano, el café, el cacao, el orito, los cítricos, la caña de azúcar y la quinua. En los últimos años se ha incrementado la variedad de productos orgánicos que, poco a poco, van ganando prestigio y abriendo sus mercados, como los camarones, el mango, la sábila, el maní e incluso las flores de exportación, todos ellos orientados principalmente a los mercados de EEUU, Unión Europea y Japón.

La hipótesis nula de esta investigación señala que la demanda y consumo de productos orgánicos en el cantón Riobamba no dependen del nivel de escolaridad ni del nivel de ingresos de su población, para su contraste se empleó un modelo de regresión lineal múltiple para determinar la significancia estadística de estas variables. Los resultados del modelo se muestran en la Tabla 1.

Tabla 1: Estadística de regresión

\begin{tabular}{|l|l|}
\hline Coeficiente de correlación múltiple & 0.238 \\
\hline Coeficiente de determinación $\mathrm{R}^{2}$ & 0.057 \\
\hline $\mathrm{R}^{2}$ ajustado & 0.041 \\
\hline Error Típico & 0.407 \\
\hline
\end{tabular}

Tabla 2: Modelo de regresión

\begin{tabular}{|l|c|c|c|l|l|}
\hline & Suma de cuadrados & Grados de libertad & Media cuadrática & $F$ & Sig. \\
\hline Regresión & 3740 & 6 & 0.623 & 3.763 & 0.001 \\
\hline Residual & 62455 & 377 & 0.166 & & \\
\hline Total & 66185 & 383 & & & \\
\hline
\end{tabular}

Las variables predictivas consideradas en este modelo explican únicamente el $4,1 \%$ del consumo de productos orgánicos, las variables que tiene un efecto significativo $(p<0,05)$ en la población, son el sexo de la personas y el lugar de compra de los productos orgánicos. Estos resultados los podemos comprobar en función de los coeficientes presentados en la Tabla 3. En base a estos resultados aceptamos la hipótesis nula ya que se demuestra estadísticamente que el nivel de ingresos y el nivel de educación de la población del cantón Riobamba no influye sobre el consumo de productos orgánicos. 
El comportamiento diferente en el consumo productos orgánicos entre hombres y mujeres se debe a que el $81,40 \%$ de la población de mujeres respondió que consume productos orgánicos y únicamente el $67,68 \%$ de los hombres tuvo esta afirmación, muy probablemente debido a que las mujeres son quienes realizan las compras de alimentos y se preocupan por el bienestar de la familia. El lugar de compra de los productos orgánicos facilita el acceso y la difusión de la variedad de productos disponibles para su consumo. La compra de productos en supermercados es aun bajo por falta de oferta con valor diferenciador y a precios accesibles para la mayor parte de la población de este cantón.

Tabla 3: Coeficientes estadísticos

\begin{tabular}{|c|c|c|c|c|c|}
\hline \multirow{2}{*}{ Modelo } & \multicolumn{2}{|c|}{ Coeficientes no estandarizados } & \multirow{2}{*}{$\begin{array}{c}\text { Coeficientes } \\
\text { tipificados }\end{array}$} & \multirow{2}{*}{$t$} & \multirow{2}{*}{ Sig. } \\
\hline & $B$ & Error típico & & & \\
\hline (Constante) & 1.421 & 0.17 & - & 8.339 & 0.000 \\
\hline Edad & 0.001 & 0.003 & 0.023 & 0.445 & 0.657 \\
\hline Sexo & -0.141 & 0.049 & -0.149 & -2.901 & 0.004 \\
\hline Nivel de educación & -0.003 & 0.040 & -0.004 & -0.073 & 0.942 \\
\hline Ingresos Mensuales & -0.036 & 0.024 & -0.077 & -1.456 & 0.146 \\
\hline $\begin{array}{l}\text { Beneficios de consumo de } \\
\text { productos orgánicos }\end{array}$ & -0.012 & 0.022 & -0.028 & -0.551 & 0.582 \\
\hline $\begin{array}{l}\text { Lugar de compra de } \\
\text { productos orgánicos }\end{array}$ & 0.052 & 0.015 & 0.170 & 3.388 & 0.001 \\
\hline
\end{tabular}

\section{DISCUSION}

La producción mundial de productos agrícolas orgánicos, es aún una actividad marginal que en la actualidad comprende alrededor de 31 millones de hectáreas (tan sólo 1.85\% del total de hectáreas de suelos cultivables a nivel mundial), con producción comercial en más de 120 países, donde, debe destacarse, en los últimos seis años se incorporaron más de 20 millones de hectáreas a la producción orgánica (ODEPA, 2007). En la presente investigación, un alto porcentaje de la población afirma que conoce y consume productos orgánicos y solo una quinta parte de la misma no los consume por diferentes razones lo que a nuestro criterio se convierte en una gran oportunidad para el desarrollo de este mercado en crecimiento con una alta demanda potencial. La agricultura orgánica se encuentra en crecimiento y de recibir apoyo externo en forma de créditos, asistencia, consolidación del mercado tanto el interno como el de exportación, e incluso compensaciones por los servicios ambientales que produce la conservación de la diversidad genética, protección de recursos como el suelo y el agua, se podrá asistir en los próximos años a un aumento importante del sector (Camacho et al., 2015). En este sentido (Biao, 2008) indica que el sector orgánico en el Reino Unido y en gran parte de Europa continental está en auge. En muchas áreas hay una ola de conversiones agrícolas cuya escala no se ha visto antes. La demanda orgánica está creciendo más rápido que la oferta interna y un sector de la agricultura que durante muchos años fue marginado se está volviendo cada vez más importante, Según ProChile, se estima que en EE.UU el mayor crecimiento de la demanda de productos orgánicos lo tendrían los condimentos, mientras que las frutas y vegetales frescos seguirían liderando el sector (ODEPA, 2007). Por otro lado los mercados orgánicos danés y británico son mercados de alimentos orgánicos industrializados y concentrados, basados en una alta proporción de alimentos importados, en algunos casos altamente procesados, y unidades de producción, procesamiento y distribución a gran escala (Wier, O’Doherty Jensen, K., Anderson, y Millock, 2008).

Más de la mitad de la población del cantón Riobamba cuenta con ingresos mensuales por familia que les permiten cubrir el costo de la canasta básica familiar. Al respecto Vasco et al., (2015), sugieren que los consumidores de alimentos ecológicos tienen un nivel educativo y un poder adquisitivo considerablemente más alto que la media nacional, nuestros resultados difieren ya que en el caso de Riobamba el consumo de productos orgánicos no depende del nivel de ingreso ni del grado de educación de su población determinado a través del modelo de regresión lineal $(p>0,05)$. En este mismo sentido (Smith et al., 2009), indican que un efecto de ingreso positivo significativo es importante para demostrar que la demanda de alimentos orgánicos puede ser dependiente del ingreso. También Moreira (2016), indica que el consumo de alimentos orgánicos está correlacionado con el ingreso anual y la formación profesional, lo que lleva a pensar que estos consumidores tienen más acceso a información sobre los beneficios que conlleva el consumo de estos alimentos y los perjuicios de los alimentos tratados con pesticidas. En este contexto considero que no existe una difusión clara de los beneficios del consumo de productos orgánicos sobre la salud de los consumidores y sobre el cuidado del medio ambiente con prácticas de producción sostenibles, lo que hace que independientemente del nivel de ingreso y el nivel de educación de la población no dispongan de información para decidir su compra e incrementar el consumo de productos orgánicos en su dieta diaria. 
La principal motivación que impulsa la compra de productos orgánicos en el cantón Riobamba son las características de saludables ya que los consideran libres de químicos, y solamente una pequeña parte de la población los compra porque conservan el medio ambiente, resultado que podría ser empleado en las estrategias de promoción y difusión de los productos orgánicos para incentivar su consumo. En este sentido de acuerdo a (Wier et al., 2008), encontraron que las decisiones de compra de alimentos orgánicos están motivadas principalmente por atributos de "bienestar privado" como la frescura, el sabor y los beneficios para la salud, atributos que pueden percibirse como compatibles con la estructura de producción y ventas moderna que coinciden con nuestros hallazgos. Los atributos orgánicos más tradicionales del 'bien público', como los atributos medioambientales y de bienestar animal, la producción en pequeña escala y el suministro local, son menos compatibles con la estructura actual del mercado, pero la mayoría de los consumidores en la práctica les conceden menos prioridad. Un tercio de la población compra los productos orgánicos en ferias especiales de este tipo de productos, otro tercio en mercados populares, y solo el 13,8\% adquiere productos orgánicos en el supermercado. En este sentido de acuerdo (ODEPA, 2007), indica que a medida que la producción orgánica ha ido avanzando en Chile, se han ido estableciendo canales de comercialización desde tiend as especializadas, pasando por sitios virtuales, hasta supermercados tradicionales que han incorporado góndolas o espacios especiales para la producción orgánica, sin olvidar la "feria orgánica", establecida anualmente con el doble propósito de vender, y a la vez promover este tipo de agricultura.

La principal razón por la que se consume productos orgánicos es para mantener y mejorar la salud y por cuidar el medio ambiente en segundo lugar de los resultados analizados. Estos resultados concuerdan con los expuesto por Bartels et al., (2006), que indica que el consumidor orgánico es impulsado por tener una identidad de consumidor de orden superior, un consumidor ambientalmente consciente, así como las creencias relacionadas con los productos orgánicos (es decir, la familiaridad del producto orgánico y la confiabilidad del producto orgánico) y las normas sociales del consumo orgánico. En otras palabras, los consumidores de alimentos orgánicos son recompensados con admiración y prestigio (Zelwak, 2012). Esta tendencia se está observando en el cantón Riobamba ya que en los principales supermercados se destina una gran parte de la percha de frutas y verduras principalmente para promocionar y vender productos orgánicos con su respectivo sello de certificación. En este sentido, se puede observar que los supermercados, en comparación con otros canales de venta, tienen la ventaja de poder garantizar una alta rotación y una calidad homogénea, garantizando así la frescura, el sabor y las cualidades de apariencia de los productos orgánicos (Wier et al., 2008).De acuerdo a Flores y Andrade (2008), indican en sus resultados que el $23,9 \%$ de los encuestados respondió que la principal razón por la cual adquieren este tipo de productos es por ser "saludables". El sabor, la frescura y la ausencia de químicos le siguen en importancia pero con porcentajes muy inferiores. Los hogares ecuatorianos que adquieren productos orgánicos/agroecológicos, lo hacen porque identifican a estos productos como beneficiosos para la salud de la familia. Desde un punto de individual de acuerdo a (Aertsens, Verbeke, Mondelaers, y Huylenbroeck, 2009) las decisiones sobre el consumo de alimentos orgánicos pueden explicarse relacionando los atributos de los alimentos orgánicos con valores más abstractos como "seguridad", "hedonismo", "universalismo", "benevolencia", "estimulación", "autodirección" y "conformidad". Apelar a estos valores puede influir positivamente en las actitudes hacia el consumo de alimentos orgánicos.

De acuerdo a los resultados reportados por Tobar et al., (2013), identifican cuatro dimensiones determinantes del consumo de productos orgánicos: beneficios de la agricultura orgánica, alimentación sana, estilos de vida y atributos de los productos orgánicos. Las dimensiones obtenidas influyen en aspectos de la actitud e intención de compra por parte de los consumidores hacia los alimentos orgánicos, y son elementos relevantes para establecer estrategias de comercialización que apoyen la masificación de los alimentos orgánicos en los consumidores (Pearson et al., 2013). En esta misma línea (RoitnerSchobesberger, Darnhofer, Somsook, y Vogl, 2008) indican que las razones principales para comprar productos orgánicos que los consumidores esperan es que sean más saludables y que los productos orgánicos sean amigables con el medio ambiente. La población compra productos agroecológicos porque son más saludables que los convencionales, es decir, aspectos como la conciencia ambiental, equidad, apoyo a los pequeños productores, entre otros, no parecen ser incentivos para optar por productos ecológicos (Vasco et al., 2015). Todos los resultados expuestos en este párrafo concuerdan con nuestros hallazgos ya que el $82,8 \%$ de la población encuestada expresa que su motivación de compra de productos orgánicos es que son más saludables y libres de químicos y en segundo plano por conservar el ambiente.

Un tercio de la población de Riobamba prioriza el precio para decidir la compra de productos orgánicos. Considerando que el nivel de producción de productos orgánicos aun es bajo los precios se mantienen elevados en comparación de los productos tradicionales, en este sentido a medida que los productores adquieren antigüedad, aumentan el área dedicada a la producción, el número de especies producidas y toman la decisión de certificarse los precios tenderán a bajar y masificarse su consumo (Camacho et al., 2015). También (Forman y Silverstein, 2012) mencionan que los alimentos orgánicos regularmente tienen 
un precio importante, los estudios agrícolas bien diseñados demuestran que los costos pueden ser competitivos y los rendimientos comparables a los de las técnicas agrícolas convencionales. Los consumidores han llegado a esperar productos y servicios más ecológicos, y las empresas han respondido, ya sea promoviendo productos nuevos que son más seguros para el medio ambiente o haciendo hincapié en los atributos que hacen que sus productos existentes sean ecológicos (Ruiz de Maya, López-López, y Munuera, 2011). En los resultados expuestos la población estaría dispuesta a pagar un precio más alto por los productos orgánicos fundamentalmente por los beneficios para su salud. Así los consumidores que no están dispuestos a pagar un mayor precio se lo puede atribuir a la falta de información sobre el tema y a las limitaciones de ingresos de la mayoría de los consumidores (Villanueva, 2016). En este sentido los resultados de (Gil, Gracia, y Sanchez, 2000) indican que los consumidores preocupados por la dieta saludable y la degradación ambiental son los más propensos a comprar alimentos orgánicos y están dispuestos a pagar una prima alta por este tipo de alimentos.

La mayor parte de la población de Riobamba compra productos orgánicos semanalmente, y estos resultados demuestran que, desde una perspectiva demográfica, los consumidores que son jóvenes, altamente educados y los estudiantes tienen más probabilidades de ser compradores regulares de alimentos orgánicos. Así (Ruiz de Maya et al., 2011) indica que los productos orgánicos representan una oportunidad potencialmente rentable para las empresas con un horizonte internacional. Por el contrario, hay una reducción en la frecuencia de compras de alimentos orgánicos entre los consumidores mayores y aquellos con niveles educativos más bajos (Pearson et al., 2013). También se debe considerar los expuesto en una experiencia Brasileña por (Blanc y Kledal, 2012). Los resultados muestran que el enfoque de salud podría ser uno de los elementos claves para animar a las personas a comprar alimentos más saludables como son los productos orgánicos. Así los productos orgánicos contienen menos residuos de plaguicidas que los productos convencionales, y consumir una dieta con productos orgánicos reduce la exposición humana a los pesticidas (Forman y Silverstein, 2012). Y este elemento también llevará a los consumidores a reaccionar positivamente hacia los alimentos verdes (Lidew et al., 2015). Es importante desarrollar estrategias de marketing, utilizando las razones para la adquisición de alimentos orgánicos tales como los problemas de salud (problemas de inocuidad) y la protección al medio ambiente, junto con el mantenimiento de los precios relativamente más alto que el precio de los productos convencionales (Villanueva, 2016). Al respecto los resultados muestran que la población considera importante la difusión de los beneficios del consumo de productos orgánicos a través de redes sociales y el internet. Una pequeña parte de la población considera que existe escasa difusión y pocos lugares de comercialización de productos orgánicos lo que se constituye en una barrera para el crecimiento de la demanda.

\section{CONCLUSIONES}

La mayor parte de la población del cantón Riobamba consume productos orgánicos y están dispuestos a pagar un precio más alto en comparación con los productos convencionales enfocados a mejorar su salud y conservar el medio ambiente en un segundo plano.

El género de las personas y el lugar de compra de productos orgánicos (ferias especiales, mercado popular y supermercados) presentan un efecto significativo en la demanda y consumo de este tipo de productos en la población del cantón Riobamba.

Finalmente, conviene destacar, que los principales problemas que limitan el crecimiento de la demanda de productos orgánicos identificados en esta investigación son la baja oferta existente, escasa difusión de productos disponibles, precios elevados, falta de garantía de productos orgánicos y corta vida útil de los productos para su consumo.

\section{REFERENCIAS}

Aertsens, J., W. Verbeke, K. Mondelaers y G. Huylenbroeck, Personal Determinants of Organic Food Consumption: A Review, British Food Journal, 111(10), 1140-1167 (2009)

Barbosa, S.D., M.B. Matteucci y otros cuatro autores, Perfil do Consumidor e Oscilações de Preços de Produtos Agroecológicos, https://doi.org/10.5216/pat.v41i4.11854, Pesquisa Agropecuária Tropical, 41(4), 602-609 (2011)

Bartels, J., M. Reinders, S. Sen, S.A. Du, Cross-Country Investigation of Organic Consumption Behavior: a Social Identification Perspective, https://doi.org/43008804, Advances in Consumer Research, 33, 212-217 (2006)

Biao, $X$, The Development of and Prospects for Organic Aquaculture Worldwide, https://doi.org/ 10.5367/000000008787167718, Outlook on Agriculture, 37(4), 255-260 (2008)

Blanc, J. y P.R. Kledal, The Brazilian Organic Food Sector: Prospects and Constraints of Facilitating the Inclusion of Smallholders, https://doi.org/10.1016/j.jrurstud.2011.10.005, Journal of Rural Studies, 28(1), 142-154 (2012)

Camacho, M., K. Arauz, N. Barboza, H.A. Martínez y J. Arias, Characterization of the Producers of Organic Vegetables Distributed in the Greater Metropolitan Area (GAM), Costa Rica, Agronomía Costarricense, 39(2), 131-142 (2015) 
Cardozo, E.R., C. Rodríguez y W. Guaita, Las Pequeñas y Medianas Empresas Agroalimentarias en Venezuela y el Desarrollo Sustentable: Enfoque basado en los Principios de Manufactura Esbelta, https://doi.org/10.4067/S071807642011000500006, Información Tecnológica, 22(5), 39-48 (2011)

Cruz, G., M. Ángel, S. Rindermann, O. Rufino y G. Tovar, Situación y Desafíos del Sector Orgánico de México, Revista Mexicana de Ciencia Agrícola, 1(4), 1-17 (2010)

Diaz, A., H. Pérez, Characterization of Organic Consumer Products in the City of Toluca, Mexico, Revista Mexicana de Agronegocios, 1178-1187 (2015)

Flores, M., A. Andrade, Consumo de Productos Orgánicos / Agroecológicos en los Hogares Ecuatorianos, Veco Ecuador, Derechos, 1, 1-86 (2008)

Forman, J. y J. Silverstein, Organic Foods, Health and Environmental Advantages and Disadvantages, https://doi.org/10.1542/peds.2012-2579, Pediatrics, 130(5), 1406-1415 (2012)

Gil, J.M., A. Gracia y M. Sanchez, Market Segmentation and Willingness to Pay for Organic Products in Spain, https://doi.org/10.1016/S1096-7508(01)00040-4, Inter. Food and Agribusiness Management Review, 3(C), $207-226$ (2000)

Lidew, L., Z. Jusoh y N. Sulaiman, Factors Affecting Green Food Practices and Consumer, Journal of Education and Social Sciences, 1(2011), 1-7 (2015)

Moreira, W., Análisis del Comportamiento del Consumidor de Alimentos Orgánicos, en las Principales Cadenas de Supermercados de la Ciudad de Guayaquil, Universidad Católica Santiago de Guayaquil, 1, 1-94 (2016)

Oficina de Estudios y Políticas Agrarios -ODEPA, Estudio del Mercado Nacional de Agricultura Orgánica, Ministerio de Agricultura, 1- 176 (2007)

Parrado, Á. e I. Montoya, Características del Consumo de Vegetales en los Estratos Socioeconómicos Medio y Alto de Bogotá, Agronomía Colombiana, 25(2), 377-382 (2007)

Patricia, L.L., J. Espejel, J. Cavazos y M. de Anda, Influencia de la Condición de Pobreza Extrema en la Intención de Compra de Productos Alimenticios Orgánicos en la Zona Urbana de Hermosillo, México, Sexto Coloquio Interdisciplinario de Doctorado - Universidad Popular Autónoma del Estado de Puebla (2012)

Pearson, D., J. Henryks, S. Parves y T. Anisimova, Organic food: Exploring Purchase Frequency to Explain Consumer Behaviour, Journal of Organic Systems, 8(2), 50-63 (2013)

Roitner-Schobesberger, B., I. Darnhofer, S. Somsook y C.R. Vogl, Consumer Perceptions of Organic Foods in Bangkok, https://doi.org/10.1016/j.foodpol.2007.09.004, Thailand. Food Policy, 33(2), 112-121 (2008)

Rueda, H., M. Gracia, M.E. Santana y J. Horbath, Los Mercados Orgánicos en México como Escenarios de Construcción Social de Alternativas, Revista Latinoamericana, 15(43), 1-18 (2016)

Ruiz de Maya, S., I. López-López y J.L. Munuera, Organic Food Consumption in Europe: International Segmentation Based on Value System Differences, https://doi.org/10.1016/j.ecolecon.2011.04.019, Ecological Economics, 70(10), 1767-1775 (2011)

Salazar, M., M. Gerardo, O. Torres y G. Martínez, Productos Orgánicos, Revista Mexicana de Agronegocios, 15(14059282), 503-513 (2011)

Salgado, L. y L. Beltrán, Factores que Influyen en el Consumo Sustentable de Productos Orgánicos en el Noroeste de México, Universidad y Ciencia, 27(3), 265-279 (2011)

Smith, T., C. Huang y B. Lin, Does Price or Income Affect Organic Choice Analysis of U.S. Fresh Produce Users, Journal of Agricultural and Applied Economics, 41(3), 731-744 (2009)

Soto, G., Agricultura Orgánica: Una Herramienta para el Desarrollo Rural Sostenible y la Reducción de la Pobreza, Memoria Del Taller, (1), 111 (2003)

Tobar, B., C. Adasme, M. Rodríguez y E. Pindado, Variables que Influyen en el Consumo de Alimentos Orgánicos en la Región del Maule, Chile, Economía Agraria y Recursos Naturales, 17(2), 49-61 (2013)

Vasco, C., G. Palacios y S. Paspuel, Socioeconomic Determinants of the Consumption of Ecological Products in Quito, 2, 23-28 (2015)

Vázquez, A., F.P. Lang, I. Peralta y F. Aguirre, Percepción del Consumidor y Productor de Orgánicos: El Mercado Ocelotl de Xalapa, Ver, México, Revista Mexicana de Agronegocios, 31, 20-29 (2012)

Velleda, N., F. Anjos y C. Lozan, La Certificación de Productos Ecológicos en España y Brasil, Agrociencia Uruguay, 18 (1), 163-171 (2014)

Villanueva, L., Preferencias del Consumidor y Disposición a Pagar por el Consumo de Tortilla de Maíz Orgánico, Estudios Sociales, 25 (47), 145-161 (2016)

Wier, M., K. O'Doherty y otros tres autores, The Character of Demand in Mature Organic Food Markets, https://doi.org/10.1016/j.foodpol.2008.01.00, Food Policy, 33(5), 406-421 (2008)

Zelwak, R., A Theory of the Conscious Class, Copenhagen Business School, 1-219 (2012) 
